\title{
Chaos and Order in the Integers Primes
}

\author{
P.M. Mazurkin ${ }^{*}$ \\ Doctor of Engineering Science, Academician of RANS, member of EANS, Volga State University of Technology, Russia \\ *Corresponding author: kaf_po@mail.ru
}

Received August 05, 2014; Revised August 08, 2014; Accepted August 10, 2014

\begin{abstract}
Statistical modeling by asymmetric waves, with variables amplitude and a half-cycle of fluctuation, dynamics of a scatter of block structure of positive part of a number of the integers prime which located in a row of 10 million natural numbers, proved emergence of three stages of growth of the left and right reference points in blocks of binary decomposition of prime numbers. These a reference point settle down on each side from the dividing line in the form of the two in the degree equal to number of the category of a binary numeral system, without unit. The first stage of critical chaos is formed by critical prime numbers 0,1 and 2 . The second stage of an accruing order begins with number 3 and comes to the end with a margin error in $1 \%$ at the 1135th category of binary notation for the left reference point. At blocks increasing on length among the integers prime by calculations after the 1135th category there comes the third stage with high definiteness of the beginning and the end of blocks of binary decomposition of positive prime numbers.
\end{abstract}

Keywords: integers prime, positive part, binary representation, blocks, a reference point, the dividing line, three stages of the critical chaos, an accruing order and high definiteness.

Cite This Article: P.M. Mazurkin, "Chaos and Order in the Integers Primes." Applied Mathematics and Physics, vol. 2, no. 4 (2014): 146-156. doi: 10.12691/amp-2-4-4.

\section{Introduction}

The nature "considers" in a binary numeral system, and the person - in decimal system. Therefore any number of the integers prime can be curtailed in a binary numeral system $[2,3,4,5]$. Then there is a geometry as most Gaussian ladder, so especially its steps without the triangular basis of this ladder (a gain of prime numbers [2]). Article purpose - to show three stages of block structure on positive part of a number of the whole prime

\begin{tabular}{|c|c|c|c|c|c|c|c|c|}
\hline$Z$ & -7 & -6 & -5 & -4 & -3 & -2 & -1 & -0 \\
\hline$P_{Z}$ & -13 & -11 & -7 & -5 & -3 & -2 & -1 & -0 \\
\hline
\end{tabular}

The design is identical to negative and positive prime. Therefore all regularities received for positive part of a row, will be fair and for other part of a series of the integers prime. Then it is possible to consider a complete asymmetric series of prime numbers $[2,4]$, and to apply results of the analysis and synthesis and to a negative half of a row.

In Table 1 the example of distribution of blocks of binary notation of prime numbers is given. In total from 10 million natural numbers failed to allocate 24 (excluding zero) block positive prime numbers. Division on blocks happens on the dividing line of growth $\pm 2^{i-1}$, where $i$ the category of system of the binary notation, corresponding to a number of natural numbers $0,1,2,3$, $4, \ldots$. Therefore the following 25 th block comes to the end numbers and to give regularities of chaos and about [1] scatter of block structure.

\section{Structure of the integers prime}

Blocks are allocated through transformation of prime numbers and the integers prime from decimal system in a binary numeral system. For a number of any power various types of structure from which in this article we allocate left (negative) and right (positive) asymmetric ranks of prime of a look are possible:

\begin{tabular}{|l|l|l|l|l|l|l|l|}
\hline 0 & 1 & 2 & 3 & 4 & 5 & 6 & 7 \\
\hline 0 & 1 & 2 & 3 & 5 & 7 & 11 & 13 \\
\hline
\end{tabular}

to $2^{25-1}=16777216$ that much more exceeds $10^{6}$. Apparently, it is possible to calculate values of boundary prime numbers if tested for ease of starting with a dividing line $2^{i-1}$.

In Table 1 the following symbols are accepted: $i$ category of a binary numeral system; $2^{i-1}$ - the dividing line between reference points of [2,3] blocks; $P_{N}^{\prime}$ - left border of the block of binary splitting number of prime numbers; $P_{N}^{\prime \prime}$ - right border of the block; $n$ - power (quantity) of prime numbers in one block; $\Delta P_{N}=P^{\prime \prime}-P^{\prime}$ - block length; $P_{Z R}^{\prime}$ - left reference point; $P_{Z R}^{\prime \prime}$ - right reference point; $p_{Z R}=P_{Z R}^{\prime \prime}-P_{Z R}^{\prime}$ - gain of [5] blocks.

In the beginning from Table 1 we will consider the power, length and a gain of blocks (Table 2). 
Table 1. Reference point, length, quantity, left and right borders of blocks of the integers prime

\begin{tabular}{|c|c|c|c|c|c|c|c|c|}
\hline \multirow{2}{*}{$\begin{array}{l}\text { Block } \\
\text { category } \\
\quad i\end{array}$} & \multirow{2}{*}{$\begin{array}{l}\text { Number } \\
\pm 2^{i-1}\end{array}$} & \multicolumn{2}{|c|}{ Block borders } & \multirow{2}{*}{$\begin{array}{c}\text { Power } \\
n_{ \pm} \text {, piece. }\end{array}$} & \multirow{2}{*}{$\begin{array}{c}\text { Block length } \\
\Delta P_{N}=P^{\prime \prime}-P^{\prime}\end{array}$} & \multicolumn{3}{|c|}{ Block reference point } \\
\hline & & $\pm P_{N}^{\prime}$ & $\pm P_{N}^{\prime \prime}$ & & & $P_{Z R}^{\prime}$ & $P_{Z R}^{\prime \prime}$ & $p_{Z R}$ \\
\hline 0 & 0.5 & 0 & 0.5 & 1 & 0.5 & 0.5 & 0.5 & 0 \\
\hline 1 & 1 & 0.5 & 1 & 1 & 0.5 & 1 & 1 & 0 \\
\hline 2 & 2 & 1 & 2 & 1 & 1 & 2 & 2 & 0 \\
\hline 3 & 4 & 2 & 3 & 1 & 1 & 3 & 5 & 2 \\
\hline 4 & 8 & 5 & 7 & 2 & 2 & 7 & 11 & 4 \\
\hline 5 & 16 & 11 & 13 & 2 & 2 & 13 & 17 & 4 \\
\hline 6 & 32 & 17 & 31 & 5 & 14 & 31 & 37 & 6 \\
\hline 7 & 64 & 37 & 61 & 7 & 24 & 61 & 67 & 6 \\
\hline 8 & 128 & 67 & 127 & 13 & 60 & 127 & 131 & 4 \\
\hline 9 & 256 & 131 & 251 & 23 & 120 & 251 & 257 & 6 \\
\hline 10 & 512 & 257 & 509 & 43 & 252 & 509 & 521 & 12 \\
\hline 11 & 1024 & 521 & 1021 & 75 & 500 & 1021 & 1031 & 10 \\
\hline 12 & 2048 & 1031 & 2039 & 137 & 1008 & 2039 & 2053 & 14 \\
\hline 13 & 4096 & 2053 & 4093 & 255 & 2040 & 4093 & 4099 & 6 \\
\hline 14 & 8192 & 4099 & 8191 & 463 & 4092 & 8191 & 8209 & 18 \\
\hline 15 & 16384 & 8209 & 16381 & 872 & 8172 & 16381 & 16411 & 30 \\
\hline 16 & 32768 & 16411 & 32749 & 1612 & 16338 & 32749 & 32771 & 22 \\
\hline 17 & 65536 & 32771 & 65521 & 3030 & 32750 & 65521 & 65537 & 16 \\
\hline 18 & 131072 & 65537 & 131071 & 5712 & 65534 & 131071 & 131101 & 30 \\
\hline 19 & 262144 & 131101 & 262139 & 10746 & 131038 & 262139 & 262147 & 8 \\
\hline 20 & 524288 & 262147 & 524287 & 20390 & 262140 & 524287 & 524309 & 22 \\
\hline 21 & 1048576 & 524309 & 1048573 & 38635 & 524264 & 1048573 & 1048583 & 10 \\
\hline 22 & 2097152 & 1048583 & 2097143 & 73586 & 1048560 & 2097143 & 2097169 & 26 \\
\hline 23 & 4194304 & 2097169 & 4194301 & 152937 & 2097132 & 4194301 & 4194319 & 18 \\
\hline 24 & 8388608 & 4194319 & 8388593 & 292705 & 4194274 & 8388593 & 8388617 & 24 \\
\hline
\end{tabular}

\section{Block Power}

By opportunities of the software environment CurveExpert-1.40 (Figure 1) has obtained equation

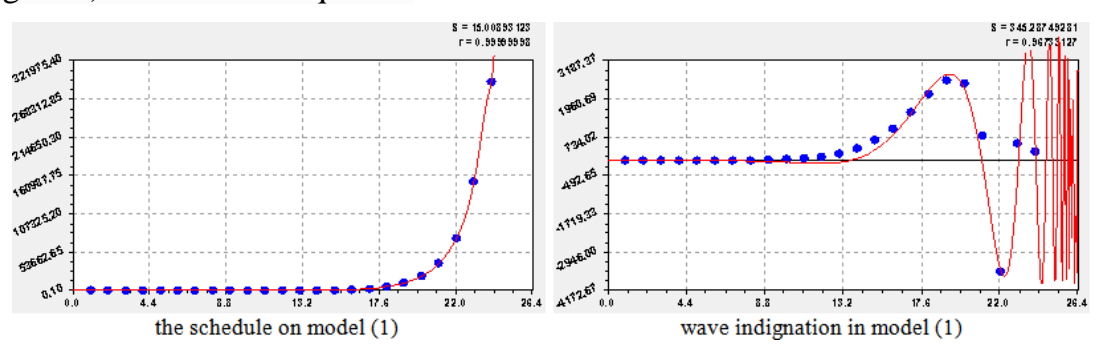

Figure 1. Schedules of power of blocks of binary decomposition of prime numbers

In Table 2 the following symbols are accepted:

$\hat{n}, \hat{\Delta}_{N}, \hat{p}_{Z R}$ - the actual values of an indicator or its difference;

$$
n=1+1,96343 \cdot 10^{-16} i^{15,35258}+A_{1} \cos \left(\pi i / p_{1}+5,78290\right)(1)
$$

$$
\begin{aligned}
& A_{1}=0,26742 i^{23,65991} \exp \left(-25,72844 i^{0,28889}\right), \\
& p_{1}=212,38011-12,45107 i^{0,88053}
\end{aligned}
$$


Table 2. Results of calculations on the revealed regularities

\begin{tabular}{|c|c|c|c|c|c|c|c|c|c|c|c|c|c|}
\hline \multirow{2}{*}{$\begin{array}{c}\text { Block } \\
\text { category } \\
i \\
\end{array}$} & \multicolumn{4}{|c|}{ Power of prime numbers (PN) in the block } & \multicolumn{5}{|c|}{ Block length } & \multicolumn{4}{|c|}{ Growth of block } \\
\hline & $\begin{array}{c}\hat{n} \\
\text { piece. }\end{array}$ & $n$, piece & $\begin{array}{c}\varepsilon, \\
\text { piece }\end{array}$ & $\Delta, \%$ & $\Delta P_{N}$ & $\hat{\Delta}_{N}$ & $\Delta_{\Delta N}$ & $\varepsilon$ & $\Delta, \%$ & $\hat{p}_{Z R}$ & $p_{Z R 1}$ & $\varepsilon$ & $\Delta, \%$ \\
\hline 0 & 1 & 1.0 & 0.000 & 0.00 & 0.5 & -0.25 & -0.25 & 0.000 & 0.00 & 0 & 0.0 & 0.000 & $\infty$ \\
\hline 1 & 1 & 1.0 & 0.002 & 0.16 & 0.5 & 0 & 0.00 & 0.001 & $\infty$ & 0 & 0.0 & 0.017 & $\infty$ \\
\hline 2 & 1 & 1.0 & 0.000 & -0.04 & 1 & 0 & 0.03 & -0.034 & $\infty$ & 0 & 0.0 & 0.007 & $\infty$ \\
\hline 3 & 1 & 1.0 & 0.001 & 0.10 & 1 & 1 & 1.00 & 0.003 & 0.26 & 2 & 2.0 & 0.005 & 0.25 \\
\hline 4 & 2 & 2.0 & -0.013 & -0.64 & 2 & 2 & 1.98 & 0.025 & 1.24 & 4 & 4.0 & 0.005 & 0.11 \\
\hline 5 & 2 & 2.0 & 0.023 & 1.14 & 2 & 6 & 5.93 & 0.075 & 1.24 & 4 & 4.0 & 0.001 & 0.04 \\
\hline 6 & 5 & 5.0 & 0.024 & 0.47 & 14 & 2 & 1.97 & 0.033 & 1.65 & 6 & 6.0 & -0.002 & -0.04 \\
\hline 7 & 7 & 7.1 & -0.069 & -0.99 & 24 & 8 & 7.95 & 0.050 & 0.63 & 6 & 6.0 & -0.001 & -0.01 \\
\hline 8 & 13 & 13.0 & -0.027 & -0.20 & 60 & 4 & 3.93 & 0.072 & 1.79 & 4 & 4.0 & 0.000 & -0.01 \\
\hline 9 & 23 & 23.0 & 0.044 & 0.19 & 120 & 8 & 7.93 & 0.066 & 0.83 & 6 & 6.0 & -0.004 & -0.07 \\
\hline 10 & 43 & 42.9 & 0.131 & 0.31 & 252 & 4 & 3.99 & 0.015 & 0.37 & 12 & 12.0 & 0.007 & 0.06 \\
\hline 11 & 75 & 75.1 & -0.094 & -0.13 & 500 & 12 & 12.02 & -0.017 & -0.14 & 10 & 10.0 & -0.010 & -0.10 \\
\hline 12 & 137 & 137.0 & -0.023 & $\begin{array}{l}-0.02 \\
\end{array}$ & 1008 & 16 & 15.97 & 0.030 & 0.19 & 14 & 14.0 & 0.005 & 0.03 \\
\hline 13 & 255 & 255.2 & -0.172 & -0.07 & 2040 & 8 & 7.93 & 0.068 & 0.85 & 6 & 6.0 & -0.010 & -0.17 \\
\hline 14 & 463 & 463.0 & -0.010 & 0.00 & 4092 & 4 & 3.97 & 0.032 & 0.80 & 18 & 18.0 & 0.005 & 0.03 \\
\hline 15 & 872 & 871.9 & 0.135 & 0.02 & 8172 & 20 & 20.00 & 0.003 & 0.01 & 30 & 30.0 & -0.006 & -0.02 \\
\hline 16 & 1612 & 1611.9 & 0.093 & 0.01 & 16338 & 46 & 45.98 & 0.023 & 0.05 & 22 & 22.0 & -0.005 & -0.02 \\
\hline 17 & 3030 & 3030.0 & 0.040 & 0.00 & 32750 & 18 & 18.06 & -0.061 & -0.34 & 16 & 16.0 & -0.002 & -0.01 \\
\hline 18 & 5712 & 5712.0 & -0.012 & 0.00 & 65534 & 2 & 2.03 & -0.026 & $\begin{array}{l}-1.30 \\
\end{array}$ & 30 & 30.0 & 0.001 & 0.00 \\
\hline 19 & 10746 & 10746.0 & -0.005 & 0.00 & 131038 & 34 & 34.06 & -0.060 & -0.18 & 8 & 8.0 & -0.007 & -0.09 \\
\hline 20 & 20390 & 20390.1 & -0.067 & 0.00 & 262140 & 4 & 3.97 & 0.034 & 0.85 & 22 & 22.0 & 0.001 & 0.00 \\
\hline 21 & 38635 & 38635.0 & 0.004 & 0.00 & 524264 & 24 & 23.98 & 0.023 & 0.09 & 10 & 10.0 & -0.009 & -0.09 \\
\hline 22 & 73586 & 73586.2 & -0.166 & 0.00 & 1048560 & 16 & 15.88 & 0.117 & 0.73 & 26 & 26.0 & -0.006 & -0.02 \\
\hline 23 & 152937 & 152936.9 & 0.067 & 0.00 & 2097132 & 20 & 19.97 & 0.032 & 0.16 & 18 & 18.0 & -0.002 & -0.01 \\
\hline 24 & 292705 & 292705.2 & -0.228 & 0.00 & 4194274 & 30 & 29.99 & 0.011 & 0.04 & 24 & 24.0 & 0.000 & 0.00 \\
\hline
\end{tabular}

Table 3. Parameters of model of dynamics of power of prime numbers on blocks of binary decomposition

\begin{tabular}{|c|c|c|c|c|c|c|c|c|c|}
\hline \multirow{3}{*}{ Number $k$} & \multicolumn{8}{|c|}{ Wavelet $y_{k}=a_{1 k} x^{a_{2 k}} \exp \left(-a_{3 k} x^{a_{4 k}}\right) \cos \left(\pi x /\left(a_{5 k}+a_{6 k} x^{a_{7 k}}\right)-a_{8 k}\right)$} & \multirow{3}{*}{ Correlation coefficient $r$} \\
\hline & \multicolumn{4}{|c|}{ amplitude (half) the fluctuations } & \multicolumn{3}{|c|}{ half-period } & shift & \\
\hline & $a_{1 k}$ & $a_{2 k}$ & $a_{3 k}$ & $a_{4 k}$ & $a_{5 k}$ & $a_{6 k}$ & $a_{7 k}$ & $a_{8 k}$ & \\
\hline 1 & 1 & 0 & 0 & 0 & 0 & 0 & 0 & 0 & \multirow{3}{*}{1.0000} \\
\hline 2 & 1.96343e-16 & 15.35258 & 0 & 0 & 0 & 0 & 0 & 0 & \\
\hline 3 & 0.26742 & 23.65991 & 25.72844 & 0.28889 & 212.38011 & -12.45107 & 0.88053 & -5.78290 & \\
\hline 4 & -0.83612 & 3.47012 & 1.54582 & 0.50396 & 22.71831 & -0.68098 & 1.08832 & -0.84556 & 0.9574 \\
\hline 5 & $2.07300 \mathrm{e}-12$ & 14.98298 & 0.80479 & 1 & 0.093755 & 0.014578 & 1.01173 & 3.45191 & 0.8257 \\
\hline 6 & $1.44368 \mathrm{e}-27$ & 35.02942 & 2.05610 & 1.00313 & -76.43455 & 7.71353 & 1.09886 & -4.54452 & 0.8099 \\
\hline 7 & 0.099221 & 2.11252 & 0.22596 & 1 & 35.64429 & -29.33368 & 0.035617 & 2.86898 & 0.8134 \\
\hline 8 & $1.10032 \mathrm{e}-31$ & 43.87807 & 2.70617 & 1.06217 & 1.47142 & 0 & 0 & 0.90717 & 0.5407 \\
\hline 9 & $1.98740 \mathrm{e} 9$ & 24.29986 & 27.11658 & 0.46684 & 1.00621 & $9.57907 e-5$ & 1 & 4.64500 & 0.5745 \\
\hline 10 & $-4.55242 \mathrm{e}-24$ & 51.05327 & 6.67279 & 0.99682 & 15.00082 & -1.04057 & 1.05894 & 0.57005 & 0.2692 \\
\hline 11 & $9.81548 \mathrm{e}-5$ & 3.82275 & 0.91056 & 0.41937 & 3.68198 & 0 & 0 & 0 & 0.5757 \\
\hline 12 & $8.27605 e-56$ & 63.56029 & 2.77650 & 1.05003 & 0.14616 & 0.010572 & 1.10484 & 3.75477 & 0.5537 \\
\hline 13 & -604.20092 & 3.45814 & 1.32718 & 1 & 139138.6 & -56060.45 & 1.00206 & -1.57171 & 0.8448 \\
\hline
\end{tabular}
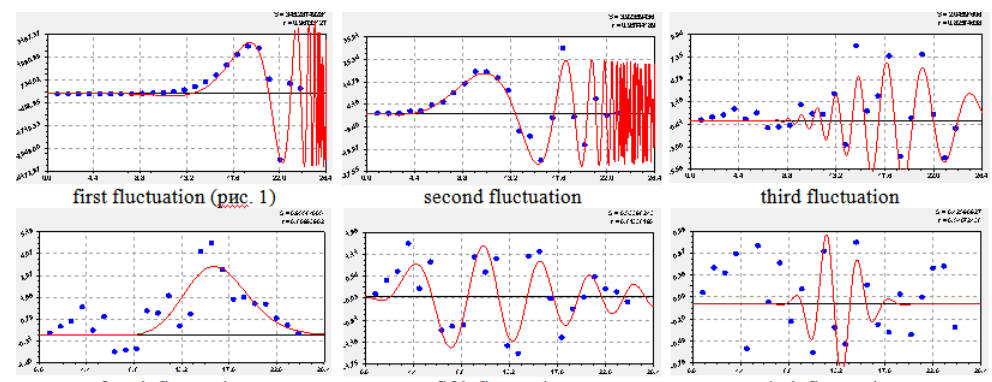

fourth fluctuation

fifth fluctuation

sixth fluctuatio
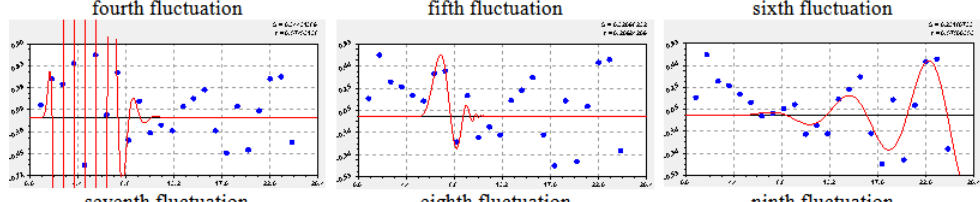

seventh fluctuation

eighth fluctuation

ninth fluctuatio
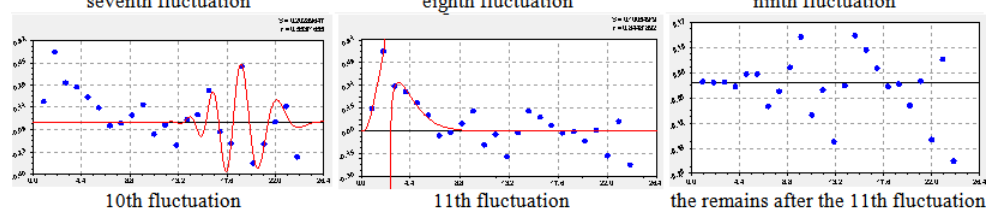

Figure 2. Schedules of model of dynamics of power of blocks of prime numbers 
Wavelet analysis [2] data of Table 2 on the power of blocks I allowed to reveal 11 fluctuations (Figure 2).

Apparently from the remains of the 11th fluctuation in figure 2, it is possible to continue identification of an asymmetric wavelet signal further. But according to Table 2 the maximum relative error is equal only $1.14 \%$.

All fluctuations have the convex schedule of change of amplitude. Therefore it is possible to assume that after any value of the category $i$ the conglomeration of fluctuations practically will come to the end.

\section{Block Length}

This parameter of a row on a difference $\hat{\Delta}_{N}=2^{i-2}-\Delta P_{N}$ receives according to Table 4 (Figure 3 ).

Table 4. Parameters of model of dynamics of length of blocks of binary decomposition of prime numbers

\begin{tabular}{|c|c|c|c|c|c|c|c|c|c|}
\hline \multirow{3}{*}{ Number $k$} & \multicolumn{8}{|c|}{ Wavelet $y_{k}=a_{1 k} x^{a_{2 k}} \exp \left(-a_{3 k} x^{a_{4 k}}\right) \cos \left(\pi x /\left(a_{5 k}+a_{6 k} x^{a_{7 k}}\right)-a_{8 k}\right)$} & \multirow{3}{*}{ Correlation coefficient $r$} \\
\hline & \multicolumn{4}{|c|}{ amplitude (half) the fluctuations } & \multicolumn{3}{|c|}{ half-period } & shift & \\
\hline & $a_{1 k}$ & $a_{2 k}$ & $a_{3 k}$ & $a_{4 k}$ & $a_{5 k}$ & $a_{6 k}$ & $a_{7 k}$ & $a_{8 k}$ & \\
\hline 1 & -0.26 & 0 & 10 & 1 & 0 & 0 & 0 & 0 & \multirow{3}{*}{0.9658} \\
\hline 2 & 0.091899 & 3.03673 & 0.67245 & 0 & 0 & 0 & 0 & 0 & \\
\hline 3 & $4.05889 \mathrm{e}-35$ & 47.66810 & 3.81780 & 0.92585 & 9.57662 & -0.31055 & 1.01433 & -1.15395 & \\
\hline 4 & $2.98139 \mathrm{e} 6$ & 49.97558 & 68.58723 & 0.28872 & 4.13328 & 0 & 0 & 0 & 0.7645 \\
\hline 5 & $-2.61845 e-97$ & 162.78702 & 14.98412 & 1.00209 & 0.16363 & 0.046893 & 1.00580 & 4.77308 & 0.6946 \\
\hline 6 & $-1.20222 \mathrm{e}-11$ & 30.03000 & 4.59708 & 1 & 1.0000025 & 0 & 0 & 0.00015 & 0.6623 \\
\hline 7 & 0.0012732 & 2.34856 & -0.014252 & 1 & 26.55273 & -1.73141 & 0.80612 & -1.77576 & 0.7015 \\
\hline 8 & 268.90813 & 3.28435 & 6.80497 & 0.28412 & 4.91589 & -0.10957 & 1.13997 & -2.51593 & 0.7909 \\
\hline 9 & 62.08714 & 3.14559 & 5.66576 & 0.31339 & 0.16015 & 0.0040389 & 1.56081 & -0.043890 & 0.7437 \\
\hline 10 & $2.87849 \mathrm{e}-27$ & 33.55907 & 2.09569 & 0.98747 & 2.02147 & -0.00030290 & 1.80201 & -2.12329 & 0.7958 \\
\hline 11 & $6.83874 \mathrm{e}-12$ & 19.36581 & 1.83802 & 1.02038 & 1.09859 & 0 & 0 & 1.80844 & 0.6968 \\
\hline 12 & -0.11112 & 2.91886 & 1.04078 & 0.78569 & 0.031913 & 0.11892 & 0.83299 & 5.43135 & 0.9134 \\
\hline
\end{tabular}
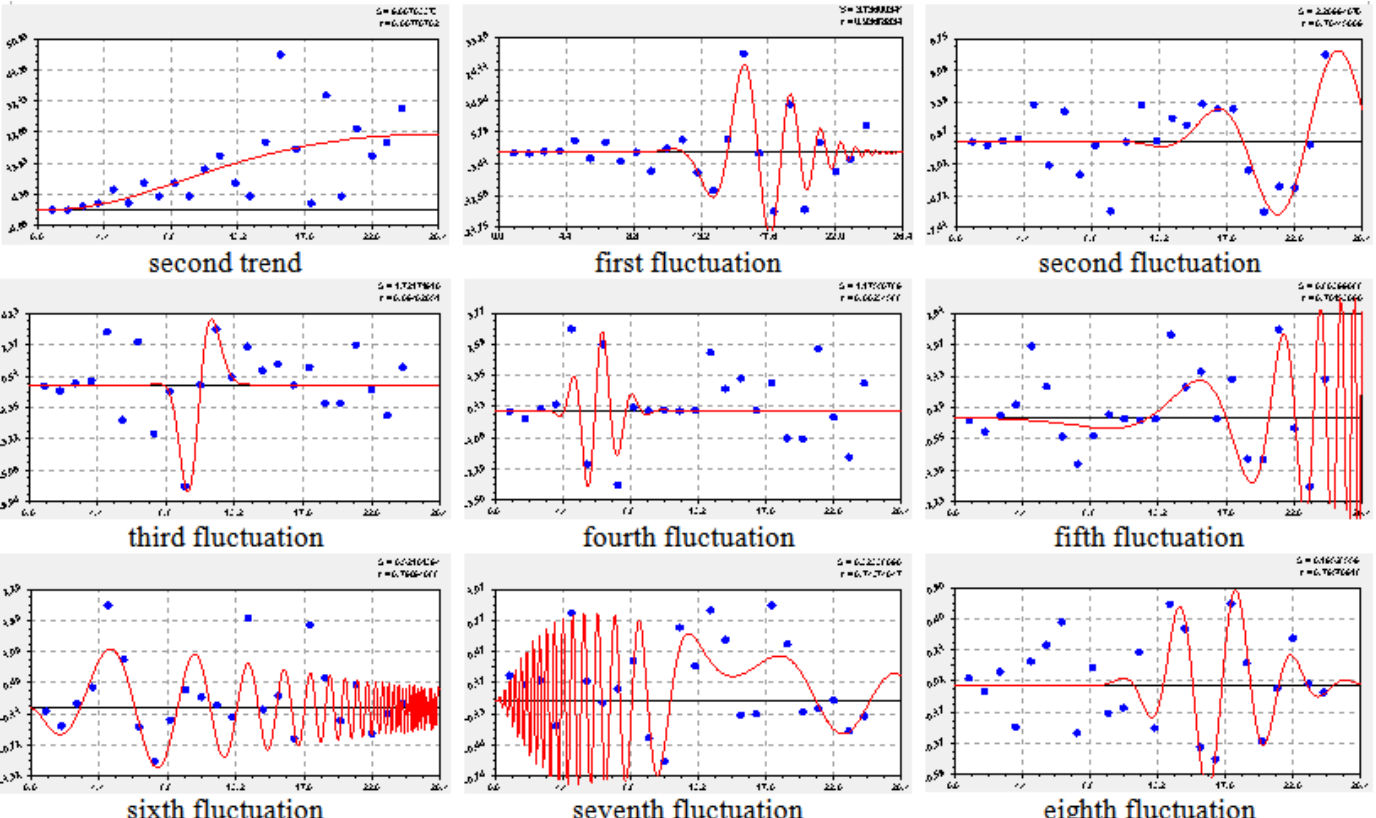

seventh fluctuation

eighth fluctuation
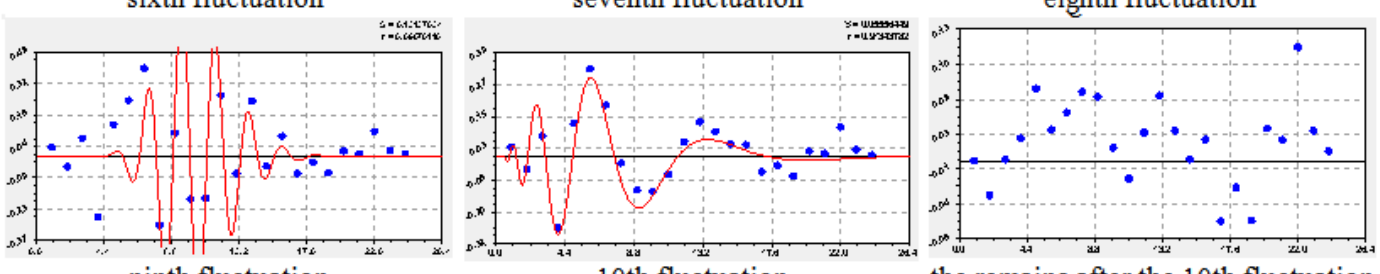

ninth fluctuation

10th fluctuation

the remains after the 10 th fluctuation

Figure 3. Schedules of members of model of dynamics of length of blocks of prime numbers

The seventh member has double growth with increase $i$ therefore block length continuously increases.

The maximum relative error of model according to Table 4 is equal $1.79 \%$ (Table 2 ).

\section{The Growth of the Block}

Between right and left reference points is the growth which is given (Figure 4, Table 5).
The first member of model according to Table 5 is the indicative law therefore the growth of blocks depending on the category of a binary numeral system grows indefinitely. The maximum relative error decreases from infinity (for critical numbers $0,1,2$ ) to $0.25 \%$ already at the category $i=3$.

With further increase in the category of a binary numeral system the error of modeling decreases. 
Table 5. Parameters of model of dynamics of a growth of blocks of binary decomposition of prime numbers

\begin{tabular}{|c|c|c|c|c|c|c|c|c|c|}
\hline \multirow{3}{*}{ Number $k$} & \multicolumn{8}{|c|}{ Wavelet $y_{k}=a_{1 k} x^{a_{2 k}} \exp \left(-a_{3 k} x^{a_{4 k}}\right) \cos \left(\pi x /\left(a_{5 k}+a_{6 k} x^{a_{7 k}}\right)-a_{8 k}\right)$} & \multirow{3}{*}{ Correlation coefficient $r$} \\
\hline & \multicolumn{4}{|c|}{ amplitude (half) the fluctuations } & \multicolumn{3}{|c|}{ half-period } & shift & \\
\hline & $a_{1 k}$ & $a_{2 k}$ & $a_{3 k}$ & $a_{4 k}$ & $a_{5 k}$ & $a_{6 k}$ & $a_{7 k}$ & $a_{8 k}$ & \\
\hline 1 & 0.32511 & 1.46430 & 0 & 0 & 0 & 0 & 0 & 0 & \multirow{3}{*}{0.9925} \\
\hline 2 & 0.030246 & 3.68196 & 1.90784 & 0.33161 & 0.33130 & 0.036944 & 1.09545 & -5.36260 & \\
\hline 3 & 0.0016402 & 3.45831 & 0.00078497 & 2.57377 & 4.17353 & 0.071430 & 1.11870 & -1.23311 & \\
\hline 4 & $4.11277 \mathrm{e}-31$ & 64.71720 & 14.31300 & 0.72260 & 1.02554 & -0.0010312 & 0.96476 & 1.01787 & 0.8875 \\
\hline 5 & -1.57504 & 1.73391 & 0.88605 & 0.78390 & 0.058654 & 0.57269 & 0.62311 & 5.98751 & 0.7881 \\
\hline 6 & $5.25201 \mathrm{e}-29$ & 34.56158 & 1.75955 & 1.03382 & $\begin{array}{l}6.66507 \\
\end{array}$ & -0.16751 & 1.01238 & 2.85875 & 0.8911 \\
\hline 7 & 0.0039274 & 3.61617 & 0.0013912 & 3.69128 & 0.87190 & 0.0011749 & 2.38852 & 2.47049 & 0.9460 \\
\hline 8 & $-1.28243 e-7$ & 5.82208 & 0.19336 & 1.00213 & 23.95592 & -0.70668 & 1.00019 & -5.73255 & 0.9159 \\
\hline 9 & $7.20607 \mathrm{e}-12$ & 13.82472 & 1.08056 & 0.97017 & 3.41558 & -0.083016 & 0.99095 & -2.06623 & 0.8852 \\
\hline 10 & $-5.82263 e-6$ & 5.04714 & 0.38637 & 1.01139 & 6.42041 & -0.15290 & 1.00217 & 0.19528 & 0.7021 \\
\hline
\end{tabular}
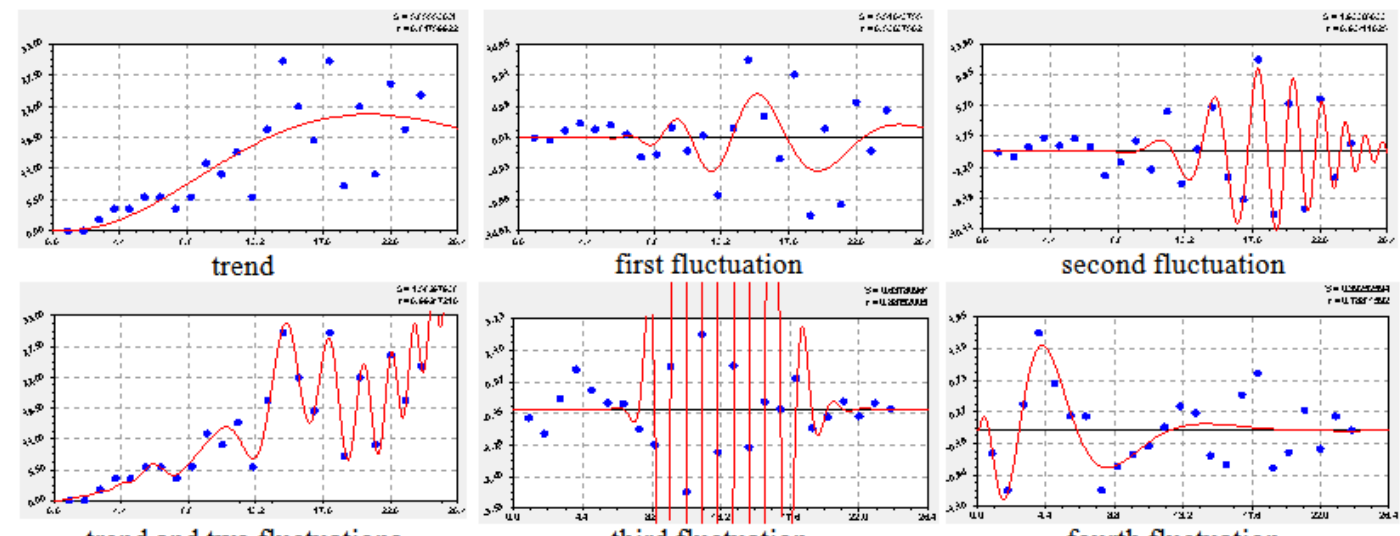

trend and two fluctuations third fluctuation

fourth fluctuation

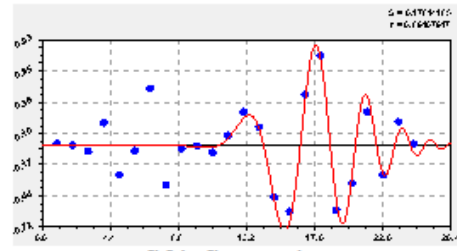

fifth fluctuation
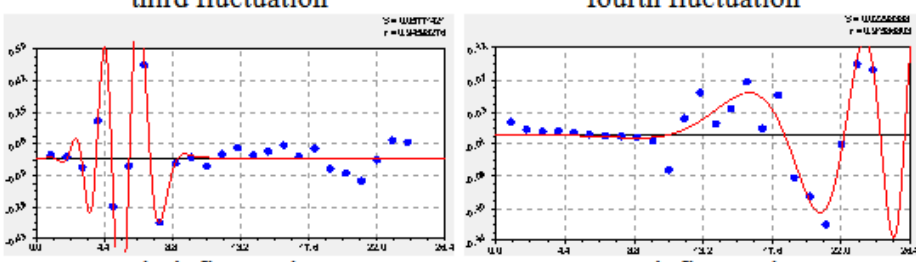

sixth fluctuation

seventh fluctuation
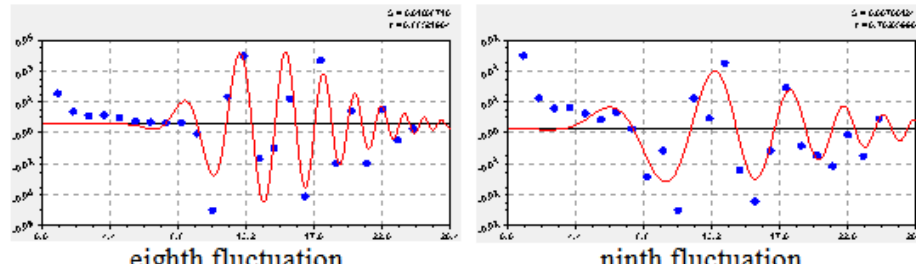

ninth fluctuation

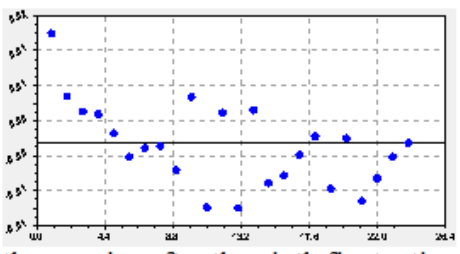

the remains after the ninth fluctuation

Figure 4. Schedules of members of model of dynamics of a growth of blocks of prime numbers

The remains after the ninth fluctuation show possibility of (2)

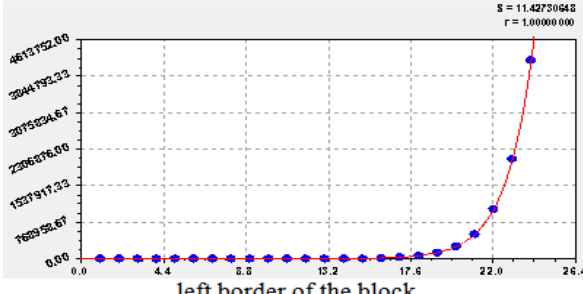

left border of the block

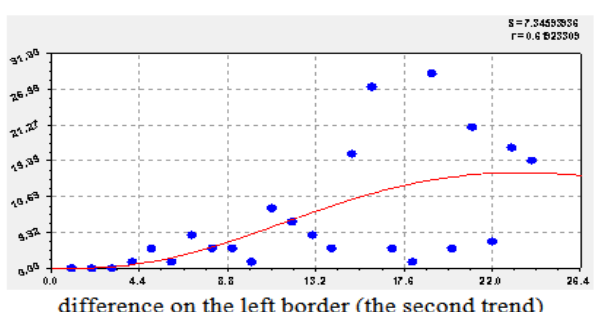

difference on the left border (the second trend)

Figure 5. Schedules of the left border of blocks of binary decomposition of prime numbers

Therefore differences on the left and right border of blocks change on formulas:

$$
\hat{P}_{\Delta}^{\prime}=P_{N}^{\prime}-2^{i-2} ; \hat{P}_{\Delta}^{\prime \prime}=2^{i-1}-P_{N}^{\prime \prime}
$$

\section{Left and Right Reference Point}

The difference between the line $2^{i-1}$ and reference points is given (Table 6):

$$
P_{Z R \Delta}^{\prime}=2^{i-1}-P_{Z R}^{\prime} ; P_{Z R \Delta}^{\prime \prime}=P_{Z R}^{\prime \prime}-2^{i-1}
$$

The dividing line is function $P_{Z R}=2^{i-1}$ of binary decomposition of prime numbers. 
Table 6. Parameters of reference points on models with wavelet signals

\begin{tabular}{|c|c|c|c|c|c|c|c|c|c|c|c|c|}
\hline \multirow[b]{2}{*}{ Block category $i$} & \multicolumn{6}{|c|}{ Left reference point (end of the previous block) } & \multicolumn{6}{|c|}{ Right reference point (of the following block) } \\
\hline & $P_{Z R}^{\prime}$ & $2^{i-1}$ & $\hat{P}_{Z R \Delta}^{\prime}$ & $P_{Z R \Delta}^{\prime}$ & $\varepsilon$ & $\Delta, \%$ & $P_{Z R}^{\prime \prime}$ & $2^{i-1}$ & $\hat{P}_{Z R \Delta}^{\prime \prime}$ & $P_{Z R \Delta}^{\prime \prime}$ & $\varepsilon$ & $\Delta, \%$ \\
\hline 0 & 0.5 & 0.5 & 0 & 0.00 & 0.000 & $\infty$ & 0.5 & 0.5 & 0 & 0.00 & 0.000 & $\infty$ \\
\hline 1 & 1 & 1 & 0 & 0.00 & 0.000 & $\infty$ & 1 & 1 & 0 & 0.03 & -0.029 & $\infty$ \\
\hline 2 & 2 & 2 & 0 & 0.00 & 0.001 & $\infty$ & 2 & 2 & 0 & -0.01 & 0.007 & $\infty$ \\
\hline 3 & 3 & 4 & 1 & 1.00 & 0.000 & -0.03 & 5 & 4 & 1 & 1.01 & -0.005 & -0.51 \\
\hline 4 & 7 & 8 & 1 & 1.00 & 0.001 & 0.11 & 11 & 8 & 3 & 2.99 & 0.013 & 0.42 \\
\hline 5 & 13 & 16 & 3 & 2.99 & 0.009 & 0.29 & 17 & 16 & 1 & 0.99 & 0.007 & 0.67 \\
\hline 6 & 31 & 32 & 1 & 1.01 & -0.010 & -0.97 & 37 & 32 & 5 & 5.01 & -0.010 & -0.21 \\
\hline 7 & 61 & 64 & 3 & 2.99 & 0.012 & 0.41 & 67 & 64 & 3 & 2.99 & 0.012 & 0.39 \\
\hline 8 & 127 & 128 & 1 & 1.00 & -0.004 & -0.39 & 131 & 128 & 3 & 3.01 & -0.011 & -0.38 \\
\hline 9 & 251 & 256 & 5 & 5.00 & -0.001 & -0.03 & 257 & 256 & 1 & 0.99 & 0.010 & 1.04 \\
\hline 10 & 509 & 512 & 3 & 3.00 & 0.001 & 0.02 & 521 & 512 & 9 & 9.01 & -0.013 & -0.15 \\
\hline 11 & 1021 & 1024 & 3 & 3.00 & 0.003 & 0.09 & 1031 & 1024 & 7 & 7.00 & 0.000 & 0.01 \\
\hline 12 & 2039 & 2048 & 9 & 9.01 & -0.007 & -0.07 & 2053 & 2048 & 5 & 5.01 & -0.015 & -0.30 \\
\hline 13 & 4093 & 4096 & 3 & 3.00 & 0.003 & 0.10 & 4099 & 4096 & 3 & 3.01 & -0.007 & -0.23 \\
\hline 14 & 8191 & 8192 & 1 & 1.00 & -0.001 & -0.06 & 8209 & 8192 & 17 & 17.01 & -0.006 & -0.04 \\
\hline 15 & 16381 & 16384 & 3 & 2.99 & 0.005 & 0.17 & 16411 & 16384 & 27 & 26.99 & 0.006 & 0.02 \\
\hline 16 & 32749 & 32768 & 19 & 19.00 & -0.004 & -0.02 & 32771 & 32768 & 3 & 2.99 & 0.015 & 0.49 \\
\hline 17 & 65521 & 65536 & 15 & 14.99 & 0.006 & 0.04 & 65537 & 65536 & 1 & 1.00 & 0.004 & 0.36 \\
\hline 18 & 131071 & 131072 & 1 & 1.00 & -0.003 & -0.35 & 131101 & 131072 & 29 & 28.99 & 0.009 & 0.03 \\
\hline 19 & 262139 & 262144 & 5 & 5.00 & 0.002 & 0.04 & 262147 & 262144 & 3 & 2.99 & 0.006 & 0.21 \\
\hline 20 & 524287 & 524288 & 1 & 1.01 & -0.005 & -0.53 & 524309 & 524288 & 21 & 21.01 & -0.008 & -0.04 \\
\hline 21 & 1048573 & 1048576 & 3 & 3.00 & 0.002 & 0.06 & 1048583 & 1048576 & 7 & 7.00 & 0.004 & 0.06 \\
\hline 22 & 2097143 & 2097152 & 9 & 9.00 & 0.002 & 0.02 & 2097169 & 2097152 & 17 & 17.01 & -0.014 & -0.08 \\
\hline 23 & 4194301 & 4194304 & 3 & 3.00 & 0.001 & 0.02 & 4194319 & 4194304 & 15 & 15.00 & 0.000 & 0.00 \\
\hline 24 & 8388593 & 8388608 & 15 & 15.00 & 0.005 & 0.03 & 8388617 & 8388608 & 9 & 9.01 & -0.008 & -0.09 \\
\hline
\end{tabular}

\section{Left Reference Point of the Block} 6.

Parameters of model are given in Table 7 and in figure

By computing opportunities of the program environment the first four components gave a formula:

$$
\begin{gathered}
P_{Z R \Delta}^{\prime}=2^{i-1}-P_{Z R}^{\prime}=P_{1}^{\prime}+P_{2}^{\prime}+P_{3}^{\prime}+P_{4}^{\prime} \\
P_{1}^{\prime}=831547,59 i^{5,66128} \exp \left(-16,89700 i^{0,17547}\right),
\end{gathered}
$$

$P_{2}^{\prime}=A_{1} \cos \left(\pi i / p_{1}-3,60887\right)$,

$A_{1}=2,05698 \cdot 10^{-26} i^{34,30024} \exp \left(-2,12259 i^{0,99190}\right)$

$p_{1}=0,016843+0,010942 i^{1,01130}$,

$P_{3}^{\prime}=A_{2} \cos \left(\pi i / p_{2}+2,41486\right)$,

$A_{2}=324459,09 i^{5,65183} \exp \left(-17,16280 t^{0,16486}\right)$,

$p_{2}=1,25666, P_{4}^{\prime}=A_{3} \cos \left(\pi i / p_{3}+2,70691\right)$,

$A_{3}=3,08217 \cdot 10^{-28} i^{32,14331} \exp \left(-1,04691 t^{1,13505}\right)$,

$p_{3}=2,73007$

\begin{tabular}{|c|c|c|c|c|c|c|c|c|c|}
\hline \multirow{3}{*}{ Number $k$} & \multicolumn{8}{|c|}{ Wavelet $y_{k}=a_{1 k} x^{a_{2 k}} \exp \left(-a_{3 k} x^{a_{4 k}}\right) \cos \left(\pi x /\left(a_{5 k}+a_{6 k} x^{a_{7 k}}\right)-a_{8 k}\right)$} & \multirow{3}{*}{ Correlation coefficient $r$} \\
\hline & \multicolumn{4}{|c|}{ amplitude (half) the fluctuations } & \multicolumn{3}{|c|}{ half-period } & shift & \\
\hline & $a_{1 k}$ & $a_{2 k}$ & $a_{3 k}$ & $a_{4 k}$ & $a_{5 k}$ & $a_{6 k}$ & $a_{7 k}$ & $a_{8 k}$ & \\
\hline 1 & 831547.59 & 5.66128 & 16.89700 & 0.17547 & 0 & 0 & 0 & 0 & \multirow{4}{*}{0.9910} \\
\hline 2 & $2.05698 \mathrm{e}-26$ & 34.30024 & 2.12259 & 0.99190 & 0.016843 & 0.010942 & 1.01130 & 3.60887 & \\
\hline 3 & 324459.09 & 5.65183 & 17.16280 & 0.16486 & 1.25666 & 0 & 0 & -2.41486 & \\
\hline 4 & $3.08217 \mathrm{e}-28$ & 32.14331 & 1.04691 & 1.13505 & 2.73007 & 0 & 0 & -2.70691 & \\
\hline 5 & 3.11568e-15 & 57.22943 & 11.41855 & 0.99988 & 1.08719 & -0.0029275 & 1.04638 & 0.92670 & 0.6674 \\
\hline 6 & 1.77192e-6 & 9.55141 & 0.77276 & 1 & 0.97889 & 0 & 0 & 2.26458 & 0.9276 \\
\hline 7 & 0.48972 & 0.24899 & 0.15647 & 1 & 1.55376 & 0.0015773 & 1 & 0.090174 & 0.7808 \\
\hline 8 & 0.029481 & 1.60174 & 0.00043664 & 3.77431 & 5.06626 & 0 & 0 & 1.93703 & 0.8713 \\
\hline 9 & 0.024580 & 3.31001 & 0.74262 & 0.99595 & 1.21523 & 0 & 0 & 0.95532 & 0.7167 \\
\hline 10 & -0.0011051 & 3.51433 & 0.77059 & 0.75659 & 1.94033 & 0 & 0 & 0.75824 & 0.5097 \\
\hline 11 & 1.63085 & 2.29086 & 2.64368 & 1 & 4.62020 & -0.48721 & 1 & 0.23623 & 0.5762 \\
\hline 12 & $2.21044 \mathrm{e}-15$ & 17.98213 & 1.29568 & 0.96622 & 1.13230 & 0.0073134 & 0.98690 & 2.15391 & 0.8493 \\
\hline 13 & 0.00010785 & 2.62564 & 0.15826 & 0.92031 & 8.78152 & -0.064854 & 1.07275 & -2.99571 & 0.7819 \\
\hline 14 & $9.71548 \mathrm{e}-5$ & 3.05036 & 0.21051 & 1.00997 & 3.96774 & -0.043977 & 1.01561 & 3.14188 & 0.8466 \\
\hline
\end{tabular}

Table 7. Parameters of dynamics of the left reference point of prime numbers on blocks of binary decomposition 

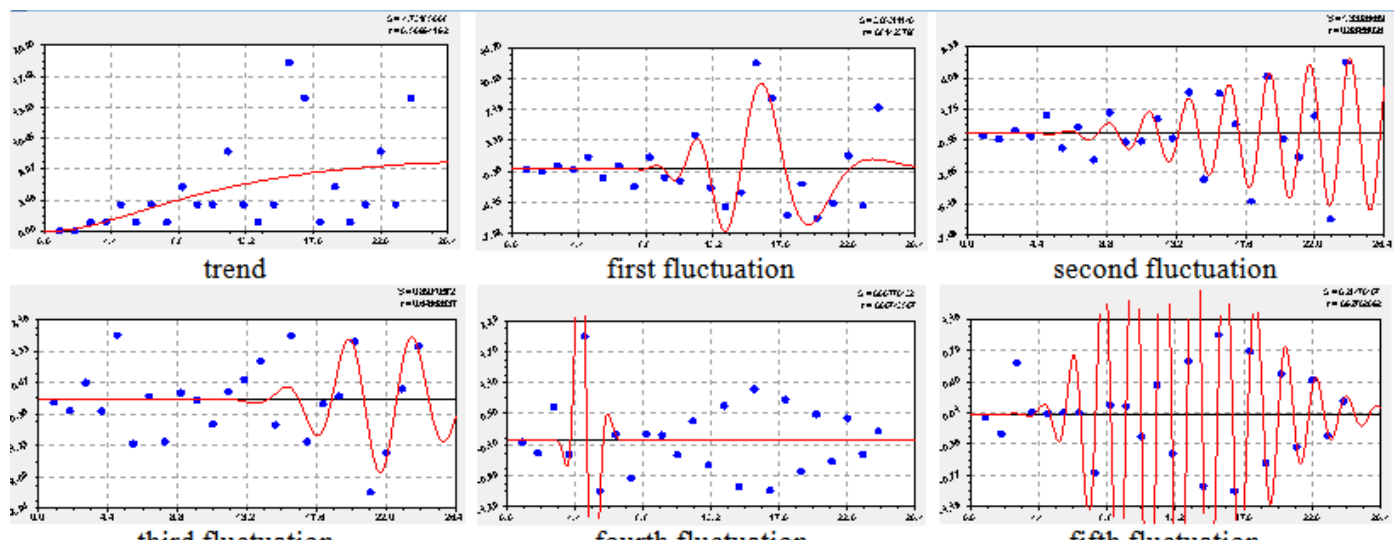

fourth fluctuation

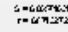
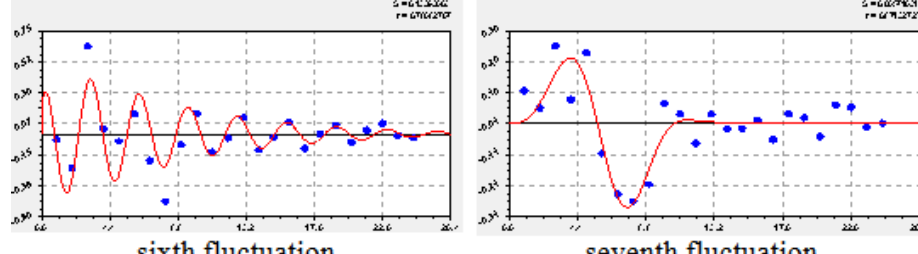

seventh fluctuation

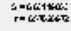

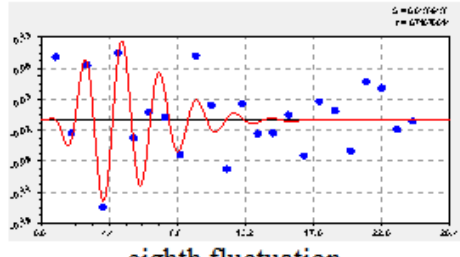

eighth fluctuation
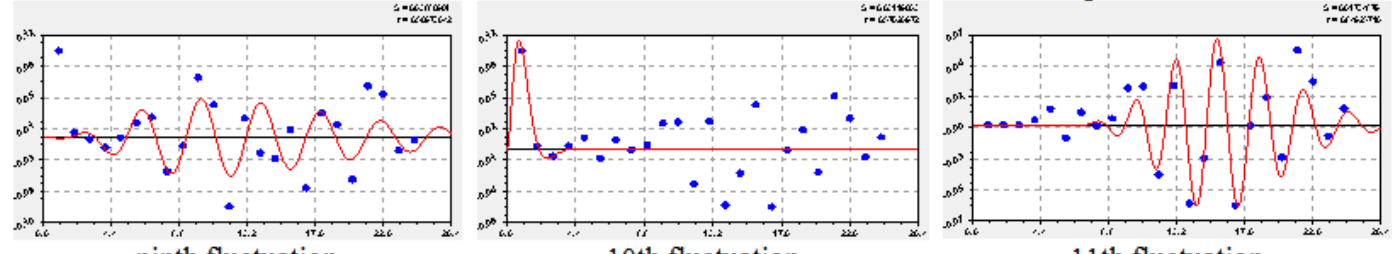

10th fluctuation
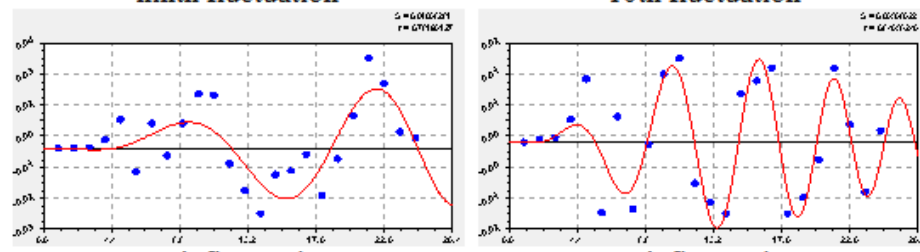

11th fluctuation

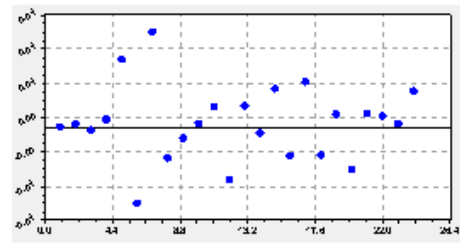

13th fluctuation

the remains after the 13 th fluctuation

Figure 6. Schedules of wave members of model of dynamics of the left reference point of blocks of prime numbers

The maximum relative error 14-membered equations according to Table 7 is equal to $0.97 \%$.

\section{Right Reference Point of the Block}

It is characterized by model with the parameters which have been set in Table 8, and schedules of components of the general model are shown in figure 7 .

The maximum relative error of the general model according to Table 8 is equal $1.04 \%$.

Table 8. Parameters of model of dynamics of the right reference point of prime numbers on blocks of binary decomposition

\begin{tabular}{|c|c|c|c|c|c|c|c|c|c|}
\hline \multirow{3}{*}{ Number $k$} & \multicolumn{8}{|c|}{ Wavelet $y_{k}=a_{1 k} x^{a_{2 k}} \exp \left(-a_{3 k} x^{a_{4 k}}\right) \cos \left(\pi x /\left(a_{5 k}+a_{6 k} x^{a_{7 k}}\right)-a_{8 k}\right)$} & \multirow{3}{*}{ Correlation coefficient $r$} \\
\hline & \multicolumn{4}{|c|}{ amplitude (half) the fluctuations } & \multicolumn{3}{|c|}{ half-period } & \multirow{2}{*}{$\begin{array}{l}\text { shift } \\
a_{8 k}\end{array}$} & \\
\hline & $a_{1 k}$ & $a_{2 k}$ & $a_{3 k}$ & $a_{4 k}$ & $a_{5 k}$ & $a_{6 k}$ & $a_{7 k}$ & & \\
\hline 1 & 0.0036181 & 4.00876 & 0.22161 & 0.94999 & 0 & 0 & 0 & 0 & \multirow{2}{*}{0.9829} \\
\hline 2 & 8.35523e-14 & 18.24021 & 0.90828 & 1.06777 & 0.060961 & 0.0077792 & 1.04229 & 0.79948 & \\
\hline 3 & 25.77481 & 86.45794 & 105.37368 & 0.32037 & 53.75898 & -38.81498 & 0.082114 & -5.85470 & 0.6057 \\
\hline 4 & 8.06279e8 & 19.66870 & 26.52183 & 0.40026 & 0.049643 & 0.018327 & 1.19235 & 3.65245 & 0.7494 \\
\hline 5 & 1448494.6 & 29.57812 & 38.21948 & 0.31731 & 1.00166 & 0 & 0 & 1.47685 & 0.8262 \\
\hline 6 & $3.83192 \mathrm{e}-13$ & 14.60945 & 0.76517 & 0.99991 & 86.17930 & -3.18500 & 0.99999 & -2.96329 & 0.6873 \\
\hline 7 & 0.0033284 & 2.19515 & 0.00055775 & 2.69399 & 9.41472 & -2.69416 & 0.32435 & -2.07606 & 0.7648 \\
\hline 8 & 0.078663 & 3.21678 & 0.63475 & 1 & 1.48895 & -0.0092734 & 1 & -2.91832 & 0.9095 \\
\hline 9 & 0.038159 & 3.64528 & 0.76216 & 1 & 29.58957 & 0.040478 & 1 & -0.47602 & 0.7512 \\
\hline 10 & $9.97608 \mathrm{e}-31$ & 39.48988 & 2.65273 & 0.99946 & 1.01431 & 0 & 0 & 1.38843 & 0.5756 \\
\hline 11 & $2.74832 \mathrm{e}-38$ & 41.19469 & 1.82273 & 1.02353 & 3.26377 & -0.040327 & 0.97737 & -3.82519 & 0.8669 \\
\hline 12 & $-1.08607 \mathrm{e}-10$ & 17.36701 & 1.97714 & 1.00404 & 3.62369 & -0.10211 & 0.99552 & 0.39702 & 0.8626 \\
\hline
\end{tabular}



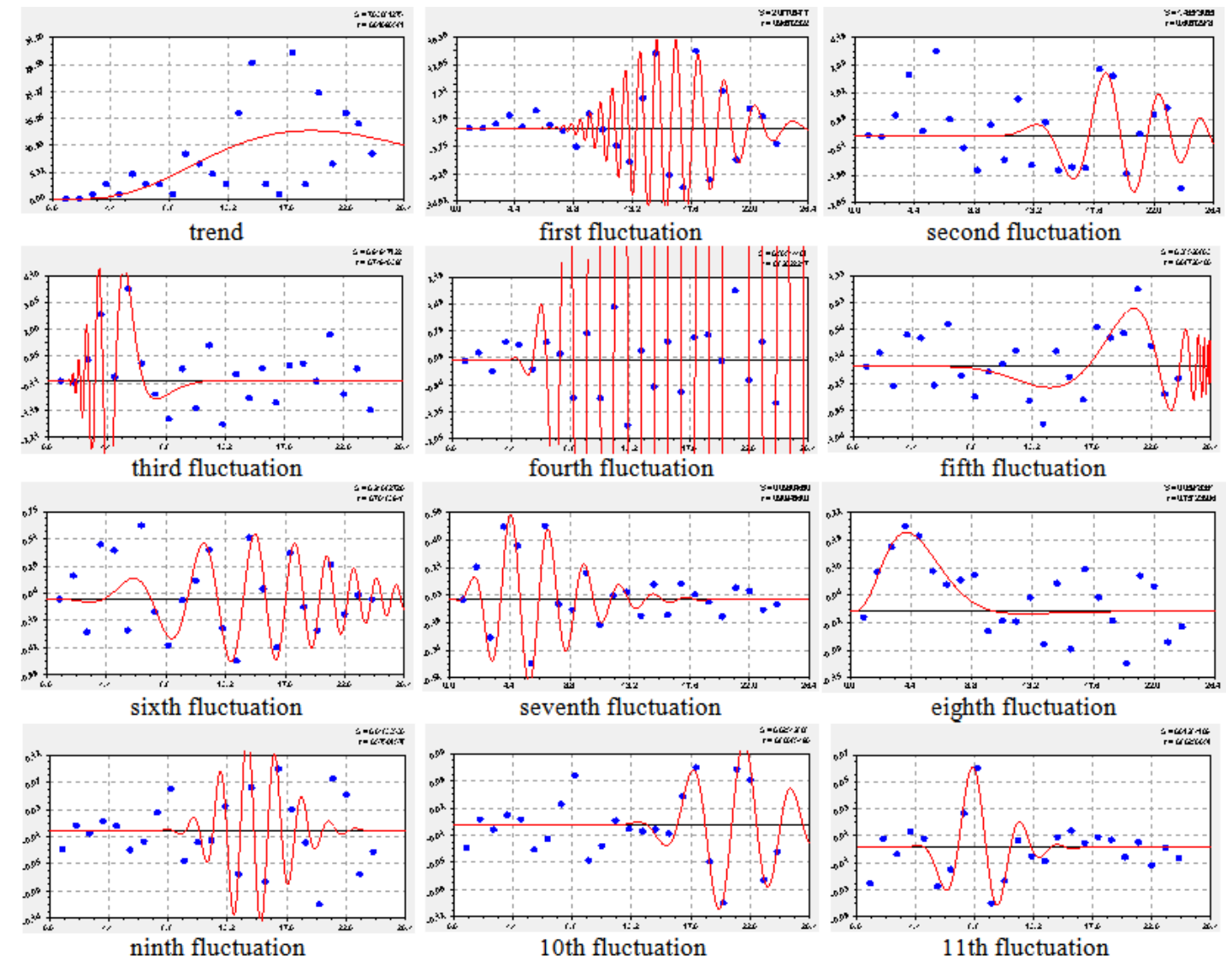

Figure 7. Schedules of wave members of model of dynamics of the right reference point of blocks of prime numbers

\section{Number of Prime Numbers as Dynamic System}

From the principles of modern informatics [1] we will consider some properties of a positive number of the integers prime. By definition, [[1], page 5] "Dynamic system is a mathematical abstraction, intended for describing and studying systems, evolving over time". Number $j$ of a prime number in a complete series [2,3] replaces a scale of calendar time, and the category $i$ of a binary numeral system becomes the additional scale of "time" leading to acceleration of growth of reference points at blocks of binary decomposition. Each block can be considered conditionally as a separate stage of evolution of prime numbers. However in this row there is no determination of property of: knowing a condition of system in different timepoints $j$ or $i$, we can't unambiguously predict further behavior of a row (generation) of prime numbers yet. However, movement along a number of prime always steadily therefore Kentropy (entropy Krylov-Kolmogorov-Sinai) is zero.

\section{Speed of a Run up of a Number of Prime}

The chaos in a series prime numbers is defined as behavior of dynamic system. «Dynamic chaos - the phenomenon in the theory of dynamic systems at which the behavior of nonlinear system looks casual in spite of the fact that it is defined by deterministic laws» [[1], page 7].

Then "K-entropy is a randomness and instability measure, it is connected with the average speed of a run up of relatives at the initial moment of trajectories. And K-entropy is greater, than trajectories run up quicker, i.e. than instability of trajectories is stronger and the system is more chaotic" [[1], page 6]. But, according to Table 6, the run up at critical categories $i=0,1,2$ precisely submits to the law $2^{i-1}$, and prime numbers receive values 0,5 (a rational root according to Riemann), 1 and 2. Left and right a reference point here are equal $2^{i-1}$, and after $i \geq 3$ a row runs up on reference points concerning the line $P_{Z R}=2^{i-1}$. When this condition is observed $P_{Z R}^{\prime}<P_{Z R}<P_{Z R}^{\prime \prime}$.

We will apply Hartley's formula to the dividing line $H=\log _{2} P_{Z R}$, where $H$ - entropy, a measure of uncertainty of experience, in which casual events (in our case experiment at a choice of two reference points of the block of prime numbers from natural numbers concerning the line $P_{Z R}=2^{i-1}$ ), $P_{Z R}$ - average number of possible outcomes of experiment on a choice of the left and right reference points are shown.

After transformation of a formula of Hartley we will receive $2^{H}=P_{Z R}$ or $2^{H}=2^{i-1}$. Therefore $H=i-1$, that is entropy of a number of prime is equal to the category of a binary numeral system without unit. From this it follows that entropy of positive part of a number of the integers prime increases in proportion to the category of binary decomposition of prime. The block structure of prime numbers has a definiteness measure $H=i-1$. 
From determination of entropy [1] follows that entropy - the numerical characteristic reflecting those degree of uncertainty which disappears after carrying out experience (in our case of experiment on definition of reference points of the following block of binary decomposition). In Table 6 were provided a reference point of prime according to the known list from 10 million natural numbers. It is necessary to apply check procedures to definition of reference points of the following 25th category on simplicity of natural numbers relatively $P_{Z R}^{i=25}=2^{24}$.

Then, let outcomes of experiences not equally likely and therefore let Shannon's formula is applicable

$$
h=\sum_{i=1}^{i} \log _{2}\left(1 / p_{i}\right)
$$

where $p_{i}$ - probability of the $i$-th outcome, for block structure of a number of prime numbers calculated on a formula

$$
\begin{aligned}
& p_{i}=\left(2^{i-1}-2^{i-2}\right) / 2^{i-1}=1-2^{i-2} / 2^{i-1} \\
& =1-2^{i-2-i+1}=1-2^{-1}=1-1 / 2=1 / 2
\end{aligned}
$$

Thus, irrespective of a size of the block of prime numbers, their probability is identical and equal $1 / 2$. Then Shannon's formula for blocks of binary decomposition of prime numbers will turn into expression $h=i$.

\section{Stages of Growth of a Number of Prime Numbers}

From data of Table 6 the first stage containing categories $i=0,1,2$ at which the relative error, because of division into zero, is equal to infinity is at once allocated.

Therefore it is possible to allocate three stages of growth of prime according to categories of binary notation:

1) $i=0,1,2$ - uncertain on critical prime numbers critical chaos;

2) $i=3,4,5, \ldots, i_{\Delta} \quad$ - accruing order (decreasing noncritical chaos as it is possible to reveal a statistical plait from a set finite-dimensional wavelets) to the set error $\varepsilon$ (us it is accepted $\varepsilon_{\Delta}=0.01$ );

3) $i>i_{\Delta}$ order with a certain small error, and under a condition $i \rightarrow \infty$ we will receive (4).

Number 3 is the first a pointer-point between the beginning of uncertainty and definiteness. With the further growth of prime definiteness increases. This property proceeds from the slow growth of a gain in comparison with a prime. The second pointer-point $i_{\Delta}$ is conditional border between a set of finite-dimensional wavelet signals and function of the dividing line $P_{Z R}=2^{i-1}$.

\section{Critical Chaos}

To understand dynamics of chaos at the beginning of a number of prime numbers, it is necessary to consider the center of symmetry of a number of the integers prime.
In figure 8 the schedule of the center of symmetry of the integers prime from seven points is shown. The schedule was received in the program CurveExpert-1.40 and it is unambiguously identified by simple function

$$
P_{Z}=Z, Z=-3,-2,-1,0,1,2,3
$$

The same proportionality is observed at the power of couples whole prime numbers $n=1 \vee 2 \vee 3$ or the total number of members of a number of integers prime $2 n+1=3,5,7$. Thus, in the center of symmetry prime numbers (their quantities also prime numbers) coincide with values of elements of a scale of integers.

The center of symmetry is invariable at any power of a number of integers prime, including and a condition $n \rightarrow \infty$. Start happens from the coefficient of proportionality equal 1 , and proceeds indefinitely.

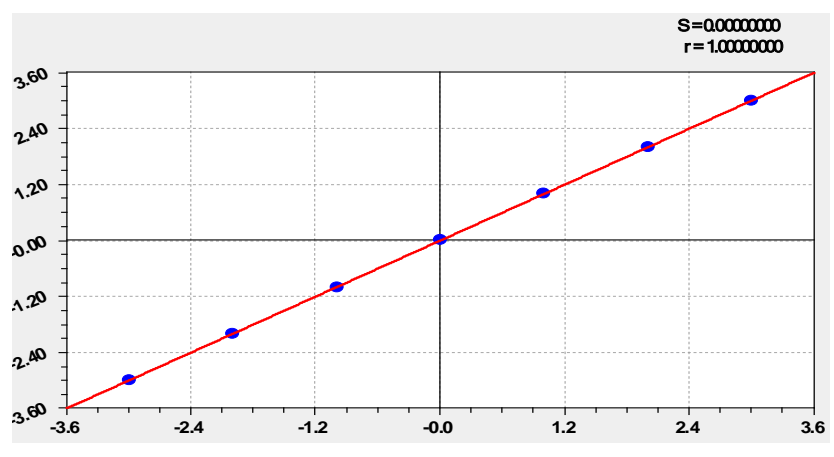

Figure 8. Center of symmetry of a number of WPN

In a spherical cover of the center of symmetry there are three fundamental physical constants (number of harmony and time) - a gold and silver proportion, and also Napier's number. From a point of singularity 0 there is difficult and while mathematically an unclear expansion to border of a kernel $[-1,0,+1]$. Then in a spherical cover $[-3,-2, \ldots, 2,3]$ there is a harmony jump through number of time (Table 9 ).

The growth of prime $p_{j}$ or integers prime $p_{Z}$ is formed when the second row moves on 1 , we will receive:

$$
p_{j}=P_{j+1}-P_{j} ; p_{Z}=P_{Z+1}-P_{Z}
$$

Physically the growth of prime is represented in the form of steps at Riemann's ladder when these steps are isolated from the most triangular case of a ladder [5].

Table 9. Growth of prime

\begin{tabular}{|c|c|}
\hline \multicolumn{2}{|c|}{ Table 9. Growth of prime } \\
\hline Prime number $P_{j}$ & Growth of prime $p_{j}$ \\
\hline 0 & 1 \\
\hline 1 & 1 \\
\hline 2 & 1 \\
\hline 2.41421 & 1.35914 \\
\hline 2.71828 & 1.61873 \\
\hline 3 & 2 \\
\hline 5 & 2 \\
\hline
\end{tabular}

We will enter the following fundamental physical constants:

- number of time (Napier's number) $e=2,71828 \ldots$;

- number of harmony (golden ratio) $\phi=(1+\sqrt{5}) / 2=1,61803 \ldots$.

- number of harmony of beauty (silver section) $1+\sqrt{2}=2,41421 \ldots$; 
- half of number of time (Napier's number) $e$ / $2=1,35914 \ldots$.

After parametrical identification of the law of achievement of a limit (or well-known law of the Weibull distribution) in the form of a formula

$$
y=y_{\max }-a \exp \left(-b x^{c}\right)
$$

was obtained (Figure 9) binomial statistical regularity

$$
p_{j \min }=2-1,02402 \exp \left(-0,00025750 P_{0,1,2,3,5}^{8,39705}\right)(11)
$$

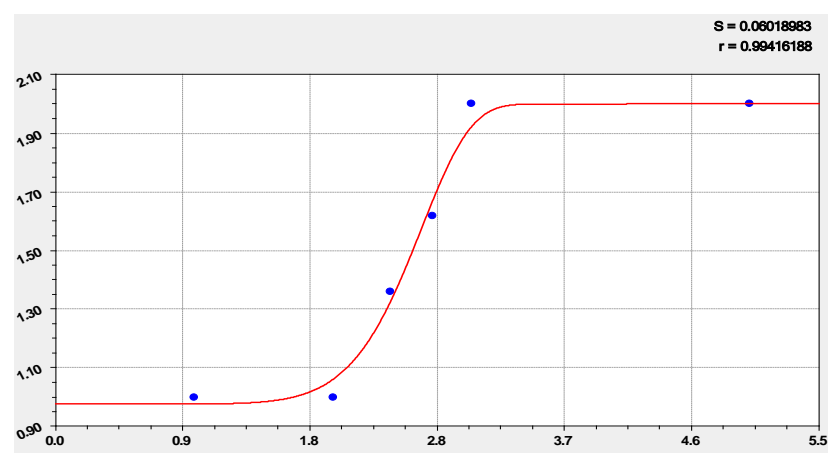

Figure 9. Gain jump from 1 to 2 in a complete series of positive prime numbers

The same formulas in a general view are valid, at the accounting of the sign "minus", and for a negative half shaft of the integers prime. Then harmony at any ranks of the whole prime numbers begins with \pm 3 .

Because of complexity of formalization of a kernel and the periphery of the center of symmetry of Gauss, and after it Riemann and other mathematics, refused the analysis of ranks, and passed to recalculation them in decimal categories.

But a number of WPN easily overcomes mathematical obstacles of two jumps (from number 0 to 1 and then from number 2 to 3 ) that in process of growth of power $n$ of couples of prime numbers there is in the beginning a recession of adequacy of identification by steady laws, and then the coefficient of correlation increases, coming nearer under a condition $n \rightarrow \infty$ again to 1 .

\section{Rising Order of the Block Structure}

During the existence the science of the information scientist saved up four main types of entropy: it is physical (in two forms - thermodynamic and statistical), technical, semantic and algorithmic entropy [1]. In this article we meant statistical physical entropy. The second stage accruing about among prime numbers is observed in the range of change of the category of a binary numeral system $i=3,4,5, \ldots, i_{\Delta}$.

For calculation $i_{\Delta}$ we will use the first component (trend) at a formula (5) of the left reference point

$$
P_{1}^{\prime}=831547,59 i^{5,66128} \exp \left(-16,89700 i^{0,17547}\right),
$$

and from parameters from Table 8 also we will write down a similar formula for the right reference point

$$
P_{1}^{\prime \prime}=0,0036181 i^{4,00876} \exp \left(-0,22161 i^{0,94999}\right) \text {. }
$$

Calculations in the program environment Excel were received maxima and borders of trends. For the left reference point received $P_{1 \max }^{\prime}=9.3521$ at $i^{*}=40$, and value $\varepsilon_{\Delta}=0.01$ turns out at $i_{\Delta}=1135$.

The right reference point changes on a difference from the dividing line $2^{i-1}$ from zero to $P_{1 \text { max }}^{\prime \prime}=13.3607$ at $i^{*}=22$, and the absolute error $\varepsilon_{\Delta}=0.01$ is observed at $i_{\Delta}=99$. Thus, the greatest interval in categories of a binary numeral system has the left reference point. Therefore with a margin error in 1\% (from a step of 1 change of a scale of categories $i$ ) the stage of an accruing order will last to $i_{\Delta}=1135$ or to natural number $2^{1134}$.

\section{Order in Block Structure of Prime Numbers}

Order - a harmonious, expected, predictable state or an arrangement something. Order - a condition of system, with sufficient degree of accuracy invariant relatively some certain shifts in space [1]. We understand a infinitedimensional number of positive prime numbers of block structure as space. Calculations for a formula (12) showed that the third stage begins with a prime number, bigger $2^{1134}$. For statistical check of the beginning of an order in positive prime numbers when wave members will be negligible, it is necessary to give block structure as Table 6 much more $i_{\Delta}=1135$.

\section{Conclusion}

Statistical modeling was carried out by asymmetric waves with variables amplitude and a fluctuation halfcycle. The loudspeaker of a run up of block structure of positive part of a number of the integers prime which is among from 10 million natural numbers, it is accurately shown on the reference points which are on each side to the dividing line $2^{i-1}$. On a set of wavelet signals emergence of three stages of growth of the left and right reference points in blocks of binary decomposition of prime is proved. These a reference point settle down on each side from the dividing line in the form of the two in the degree equal to number of the category of a binary numeral system without unit.

Therefore it is possible to allocate three stages of growth of a number of prime according to categories of binary notation:

1) $i=0,1,2$ - uncertain on critical prime numbers critical chaos;

2) $i=3,4,5, \ldots, i_{\Delta}$ - the accruing order (decreasing noncritical chaos as it is possible to reveal a statistical plait from a set finite-dimensional wavelets) to the set error $\varepsilon$ (is accepted $\varepsilon_{\Delta}=0.01$ );

3) $i>i_{\Delta}$ order with a certain small error, and under a condition $i \rightarrow \infty$ we will receive (4).

At blocks increasing on length in a positive number of the integers prime by calculations after $i_{\Delta}=1135$ there 
comes the third stage with high definiteness of the beginning and the end of blocks of binary decomposition.

\section{Reference}

[1] I.N. Beckman, "Informatics. Course of lectures." URL: http://profbeckman.narod.ru/InformLekc.htm
[2] P.M. Mazurkin. "Patterns of primes". Germany: Palmarium Academic Publishing, 2012. 280 p.

[3] P.M. Mazurkin, "Series Primes in Binary." American Journal of Applied Mathematics and Statistics, vol. 2, no. 2 (2014): 60-65.

[4] P.M. Mazurkin, "Proof the Riemann Hypothesis." American Journal of Applied Mathematics and Statistics, vol. 2, no. 1 (2014): 53-59.

[5] P.M. Mazurkin, “Increment Primes.” American Journal of Applied Mathematics and Statistics, vol. 2, no. 2 (2014): 66-72. 